\title{
HRE Finder: A Tool for Quarrying Hypoxia-Response Element in Genomic Sequences
}

\author{
Iliyas Rashid ${ }^{1,2}$, Naresh Sahebrao Nagpure ${ }^{3}$, Prachi Srivastava ${ }^{2}$, Ajey Kumar Pathak ${ }^{1 *}$, \\ Ravindra Kumar ${ }^{1}$, Vishwamitra Singh Baisvar ${ }^{1}$, Mahender Singh ${ }^{1}$ and Basdeo Kushwaha ${ }^{1}$ \\ ${ }^{1}$ Molecular Biology and Biotechnology Division, ICAR- National Bureau of Fish Genetic \\ Resources, Lucknow- 226 002, Uttar Pradesh, India \\ ${ }^{2}$ AMITY Institute of Biotechnology, AMITY University Uttar Pradesh, Lucknow-226 028, \\ Uttar Pradesh, India \\ ${ }^{3}$ Fish Genetics and Biotechnology Division, ICAR- Central Institute of Fisheries Education, \\ Mumbai-400 061, Maharashtra, India \\ *Corresponding author
}

\section{A B S T R A C T}

Keywords

Database, Hypoxia inducible factor, Hypoxia response element, Hypoxia, Transcription, Gene, Enhancer, Search algorithm.

Article Info

Accepted:

19 June 2017

Available Online:

10 July 2017
During hypoxia, the hypoxia inducible factor (HIF) binds to hypoxiaresponse elements (HRE) and induces the expression of hypoxia-inducible genes (HIG). A functionally active HRE typically contains an adjacent hypoxia ancillary sequence (HAS) in its downstream within the space of 715 nt. Here, a computational tool 'HRE Finder' is discussed using an example data set. The tool provides the ability for mining canonical HREs and predicting functional HREs in the upstream region of genes, which can be an indispensable tool for genome-wide analysis and identification of hypoxia inducible genes in vertebrates.

\section{Introduction}

Hypoxia is a condition of oxygen deficiency in the body tissues that affects proper metabolic activity, alters the expression of several genes and causes various abnormalities in different animals, ranging from invertebrates to mammals (Guillemin and Krasnow, 1997). Some genes are known to have a role in hypoxia tolerance, and their expression mechanism is induced by a transcriptional factor known as Hypoxia
Inducible Factor (HIF). HIF is a heterodimeric transcription factor composed of an HIF- $\alpha(1 \alpha, 2 \alpha$ and $3 \alpha)$ and an HIF- $\beta$ $(1 \beta, 2 \beta$ and $3 \beta)$ subunits (Wang and Semenza, 1995). A wide range of mammalian cells were well studied for the transcriptional responses to hypoxia and zebrafish have been used as a model for the study of the pathway in hypoxic condition (Smith et al., 2006; van Rooijen et al., 2011). HIF recognizes Hypoxia 
Responsible Elements (HREs), a transcription binding site (TFBs), within the promoters of a large number of genes following hypoxic induction and binds to HREs of the gene and enhances the expression of the gene (Wenger et al., 2005). HREs are normally distributed in the genome and their frequent occurrences have been reported in the enhancer or promoter region and 3' UTR of a gene (Fordel et al., 2004). The list of confirmed HIF target genes is ever expanding.

The functionally active HREs were observed in the promoter region of more than 100 mammalian genes involved in different biological processes (Manalo et al., 2005; Semenza, 2010). The frequent occurrence of functional HREs involving in transcriptional response was observed in the $1 \mathrm{~kb}$ upstream stream from the transcription start site while more than $70 \%$ hypoxia responsive genes were found without undetectable HIF-binding site in proximal promoters of a gene (Benita, 2006). Further, the frequently distributed HREs (5'-RCGTG-3') are functionally activated when its downstream contains an adjacent hypoxia ancillary sequence (HAS) in the space of 7-15 nucleotide (Kajimura et al., 2006; Liu et al., 1995).

HRE and HAS are five nucleotide motifs and their consensus patterns [(5'-RCGTG-3') and $\left.\left\{5^{\prime}-\mathrm{CA}(\mathrm{G} \mid \mathrm{C})(\mathrm{A} \mid \mathrm{G})(\mathrm{T}|\mathrm{G}| \mathrm{C})-3^{\prime}\right\}\right]$ were reported in several hypoxia-inducible genes in mammals and IGFBP1 gene in fish (Kimura et al., 2001; Kajimura et al., 2006). Approaches were applied to identify the hypoxia-inducible factor binding sites and target genes in genome-wide analysis (Greenald et al., 2015; Ortiz-Barahona et al., 2010). There are available numbers of software tools and packages like MEME Suite and MOODS (Korhonen et al., 2009; Bailey et al., 2009) for predicting conserved motifs and all types of TFBs in the noncoding genomic sequences. Unfortunately, at present, there is not even a single tool is available specifically dedicated for the finding of HRE sites and identifying functional HRE in the upstream region of the genes. Keeping this in view, a program 'HREFinder' was developed in Perl for mining all canonical HREs in the upstream sequence input by the user and predicting functional HREs.

\section{Materials and Methods}

\section{Algorithm implementation}

The widely accepted Boyer-Moore string search algorithm (Boyer and Moore, 1977) was adopted here for pattern searching in a Perl program 'HREFinder'. The pattern based algorithm is case-sensitive, hence all input sequences converted first in the upper-case string to ensure a proper match in appropriate pattern finding.

An exact match of the pattern (conserved) and approximate match with fixed number of variations between them (consensus) were applied in this algorithm. This program uses the 5'-RCGTG-3' motif to mine all canonical HREs in the query sequence and produced the result in table 1. Further, it uses the approaches of the Kimura's (2001) and Kajimura's (2006) for the identification of the functional HRE with an adjacent HAS motif, $5^{\prime}-\mathrm{CA}(\mathrm{G} \mid \mathrm{C})(\mathrm{A} \mid \mathrm{G})(\mathrm{T}|\mathrm{G}| \mathrm{C})-3$ ', in the space of 7$15 \mathrm{nt}$ of the downstream of HRE. Additionally, this program predicts all the possible canonical functional HREs with the presence of HAS consensus motif in its downstream. After a number of successful exercises, HREFinder was found is a useful tool for identification of HRE and functional HRE sites in the upstream query sequence. The download folder contains (i) a main program 'HREFinder. pl', (ii) two subdirectories 'Test Data Set' and 'Results Test Data Set' (iii) a 'readme' file, which describes the working of the program. 


\section{Preparation of test data}

A test analysis of the 'HREFinder' was initialized by preparing the query dataset. In this process, a collection of $5000 \mathrm{nt}$ (-4900 to +100 ) long upstream sequence of some reported HIG such as EPO, VEGF, IGFBP1, LdhB, HO-1, ALDOa and ENO1 from different species, like human, cattle, mouse, zebrafish, Atlantic salmon and takifugu, were used for analysis. The information on these selected genes, like gene id, gene name, gene symbol, gene location on genomic sequence, orientation, chromosome numbers and genomic sequences, was downloaded from NCBI (NCBI Resource Coordinators, 2017). A summary of the prepared dataset used for the test analysis is presented in the supplementary table 1 . The $5000 \mathrm{nt}$ long upstream sequences of each gene were parsed using the defined gene location on its respective genomic DNA with the help of an in-house developed Perl program 'upstream parser'. The upstream sequences for the genes defined on the negative orientation were parsed using reverse complement methods. The full methodology of parsing upstream from genomic sequences and mining HRE is presented in figure 1. Two types of example data files were prepared in FASTA format of original upstream, i.e. (i) a single file of all sequences, and (ii) files for the individual sequence set of the homologous genes. The other input and their corresponding output files of example dataset are stored in respective sub-directories of the tool.

\section{Results and Discussion}

This program takes an input file of sequences in FASTA format and simultaneously identifies the canonical HRE on both the strands of the query sequences and generates three output files: (i) a table of all the identified HREs, (ii) list of all possible functional HRE, and (iii) HREs distribution and their frequencies in the set of $500 \mathrm{nt}$ long consecutive fragments covering entire length of input sequences. Figure 2 illustrates the output produced by an example query of the upstream sequences set for gene VEGF. It presents canonical HRE frequencies in each $500 \mathrm{nt}$ long successive sequences covering the entire length (Figure 2-A) and predicted functional HRE (Figure 2-B). Similarly, example queries and results files for other gene sets given in table 1, are available in the respective sub-directories of online available tool. This tool is useful to the scientific community for undertaking genome-wide mining of HRE and functional HRE sites in the upstream sequences of vertebrates.

Fragments of $3-5 \mathrm{~kb}$ upstream from TSS including few hundred bases of downstream was used for the analysis of the upstream region to detect promoter and TFBs of a gene (Greenland et al., 2015; Ortiz-Barahona et al., 2010). In this process, a collection of $5000 \mathrm{nt}$ $(-4900$ to +100$)$ long upstream sequence of genes were used to prepare our test data set. The pattern based search algorithm was implemented in a web application (Rashid et $a l ., 2017)$ to analyze fishes data for functional HRE prediction and found appropriate output. Here, the algorithm is implemented in Perl program with extended analytical activity and available in form of an stand-alone downloading tool for analyzing of the genomic data of all vertebrates with a wider scope of the utilities. The HREFinder is applicable for genome-wide mining of hypoxia binding sites and predicts functional HRE in the varieties of vertebartes.

There are several tools and packages for TFBs prediction are available on the web. These tools rely upon a set of aligned promoter sequences to calculate position weight matrices (PWMs) for the identification of the conserved pattern in the no coding region of genes. 
Table.1 List of the different genes which upstream sequences were taken to prepare test dataset for analysis

\begin{tabular}{|c|c|c|c|c|c|c|c|c|}
\hline No & Animal name & GeneID & Symbol & chromosome & $\begin{array}{l}\text { Genomic } \\
\text { nucleotide } \\
\text { accession } \\
\end{array}$ & $\begin{array}{l}\text { Start position } \\
\text { on the genomic } \\
\text { accession }\end{array}$ & $\begin{array}{l}\text { End position } \\
\text { on the genomic } \\
\text { accession }\end{array}$ & orientation \\
\hline 1. & Bos taurus & 509566 & ALDOA & 25 & AC_000182.1 & 26470486 & 26483394 & minus \\
\hline 3. & Bos taurus & 280784 & EPO & 25 & AC_000182.1 & 36409504 & 36411892 & Minus \\
\hline 4. & Bos taurus & 513221 & HMOX1 & 5 & AC_000162.1 & 73980776 & 73987841 & Plus \\
\hline 6. & Bos taurus & 281275 & LDHB & 5 & AC_000162.1 & 88962610 & 88981219 & Plus \\
\hline 7. & Bos taurus & 281572 & VEGFA & 23 & AC_000180.1 & 17255515 & 17270515 & Plus \\
\hline 8. & Danio rerio & 336425 & aldoaa & 3 & NC_007114.6 & 39425126 & 39435194 & Plus \\
\hline 9. & Danio rerio & 334116 & enola & 23 & NC_007134.6 & 22729939 & 22751294 & Plus \\
\hline 10. & Danio rerio & 100004455 & epoa & 7 & NC_007118.6 & 21624135 & 21652055 & Minus \\
\hline 13. & Danio rerio & 30497 & ldhba & 4 & NC_007115.6 & 16840135 & 16846928 & Minus \\
\hline 14. & Danio rerio & 30682 & vegfaa & 16 & NC_007127.6 & 4459314 & 4488660 & Minus \\
\hline 15. & Homo sapiens & 226 & ALDOA & 16 & NC_000016.10 & 30053090 & 30070420 & Plus \\
\hline 16. & Homo sapiens & 2023 & ENO1 & 1 & NC_000001.11 & 8861000 & 8879092 & Minus \\
\hline 17. & Homo sapiens & 2056 & EPO & 7 & NC_000007.14 & 100720800 & 100723700 & Plus \\
\hline 18. & Homo sapiens & 3162 & HMOX1 & 22 & NC_000022.11 & 35381067 & 35394214 & Plus \\
\hline 19. & Homo sapiens & 3484 & IGFBP1 & 7 & NC_000007.14 & 45888360 & 45893668 & Plus \\
\hline 20. & Homo sapiens & 3945 & LDHB & 12 & NC_000012.12 & 21635342 & 21657971 & Minus \\
\hline 21. & Homo sapiens & 7422 & VEGFA & 6 & NC_000006.12 & 43770209 & 43786487 & Plus \\
\hline 28. & Mus musculus & 22339 & Vegfa & 17 & NC_000083.6 & 46016993 & 46032377 & Minus \\
\hline 29. & Salmo salar & 100380798 & aldoa & ssa03 & NC_027302.1 & 51093248 & 51105749 & Minus \\
\hline 30. & Salmo salar & 106608895 & epo & ssa07 & NC_027306.1 & 19515329 & 19529184 & Plus \\
\hline 31. & Salmo salar & 100195468 & ldhb & ssa17 & NC_027316.1 & 34597191 & 34608811 & Minus \\
\hline 32. & Salmo salar & 106584182 & LOC106584182 & $\mathrm{ssa02}$ & NC_027301.1 & 35143675 & 35159397 & Minus \\
\hline 33. & Takifugu rubripes & 101071185 & aldoa & 1 & NC_018890.1 & 8770282 & 8773425 & Plus \\
\hline 34. & Takifugu rubripes & 101069815 & eno1 & 3 & NC_018892.1 & 5889540 & 5895914 & Minus \\
\hline 35. & Takifugu rubripes & 101070079 & hmox1 & 17 & NC_018906.1 & 11058878 & 11060574 & Minus \\
\hline 36. & Takifugu rubripes & 101074885 & igfbp1 & 22 & NC_018911.1 & 14120 & 17689 & Minus \\
\hline 37. & Takifugu rubripes & 101079766 & ldhb & 18 & NC_018907.1 & 242417 & 245457 & Plus \\
\hline 38. & Takifugu rubripes & 101073237 & vegfa & 16 & NC_018905.1 & 5097117 & 5108133 & Plus \\
\hline
\end{tabular}


Fig.1 Parsing upstream sequences of genes in different orientation (A) parsing upstream from genomic sequences for the gene localized on positive strand (B) parsing upstream from genomic sequences for the gene localized on negative strand and (C) Mining HRE from both strands of the upstream sequence

A Genomic Accession NC_007127.6 (Human)
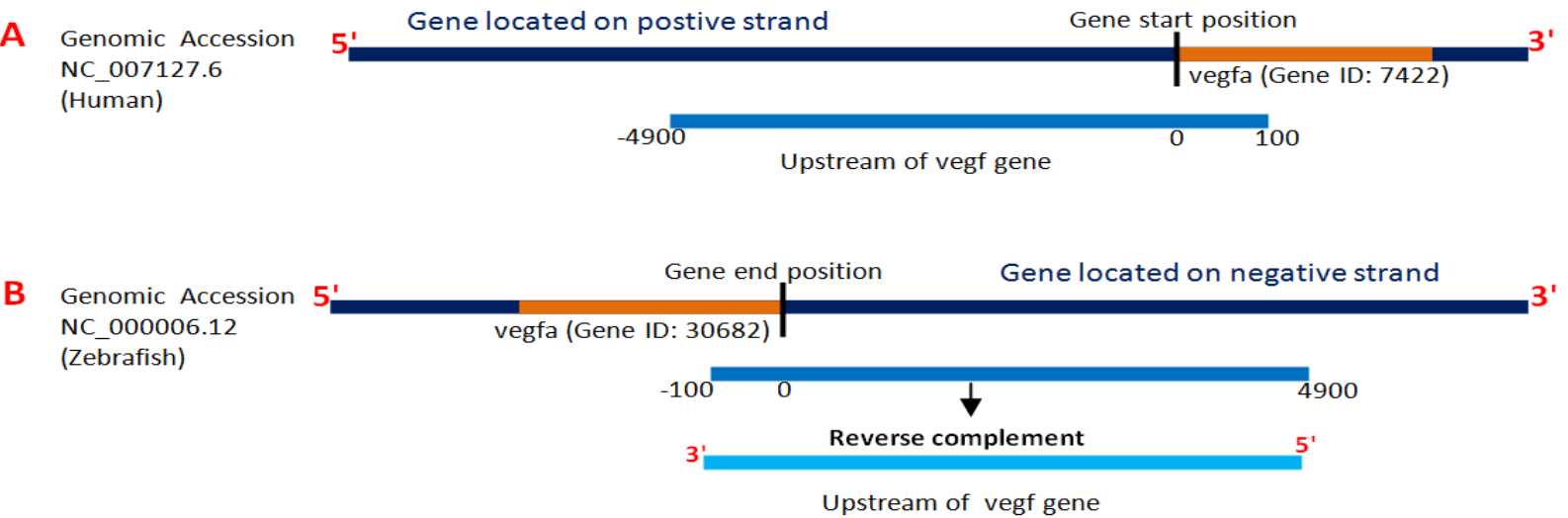

C Canonical HRE mining in upstream sequences

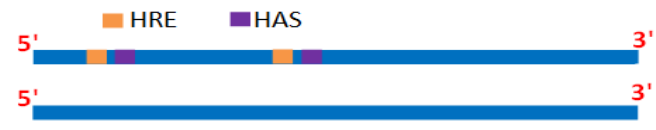
Human
Zebrafish

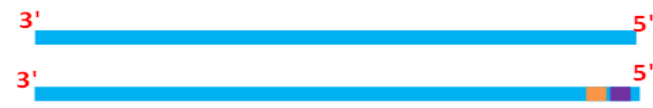

Fig.2 Output of HREFinder program for upstream VEGF gene sequences: (A) HRE frequencies in each $500 \mathrm{nt}$ long consecutive fragments, and (B) predicted functional HREs

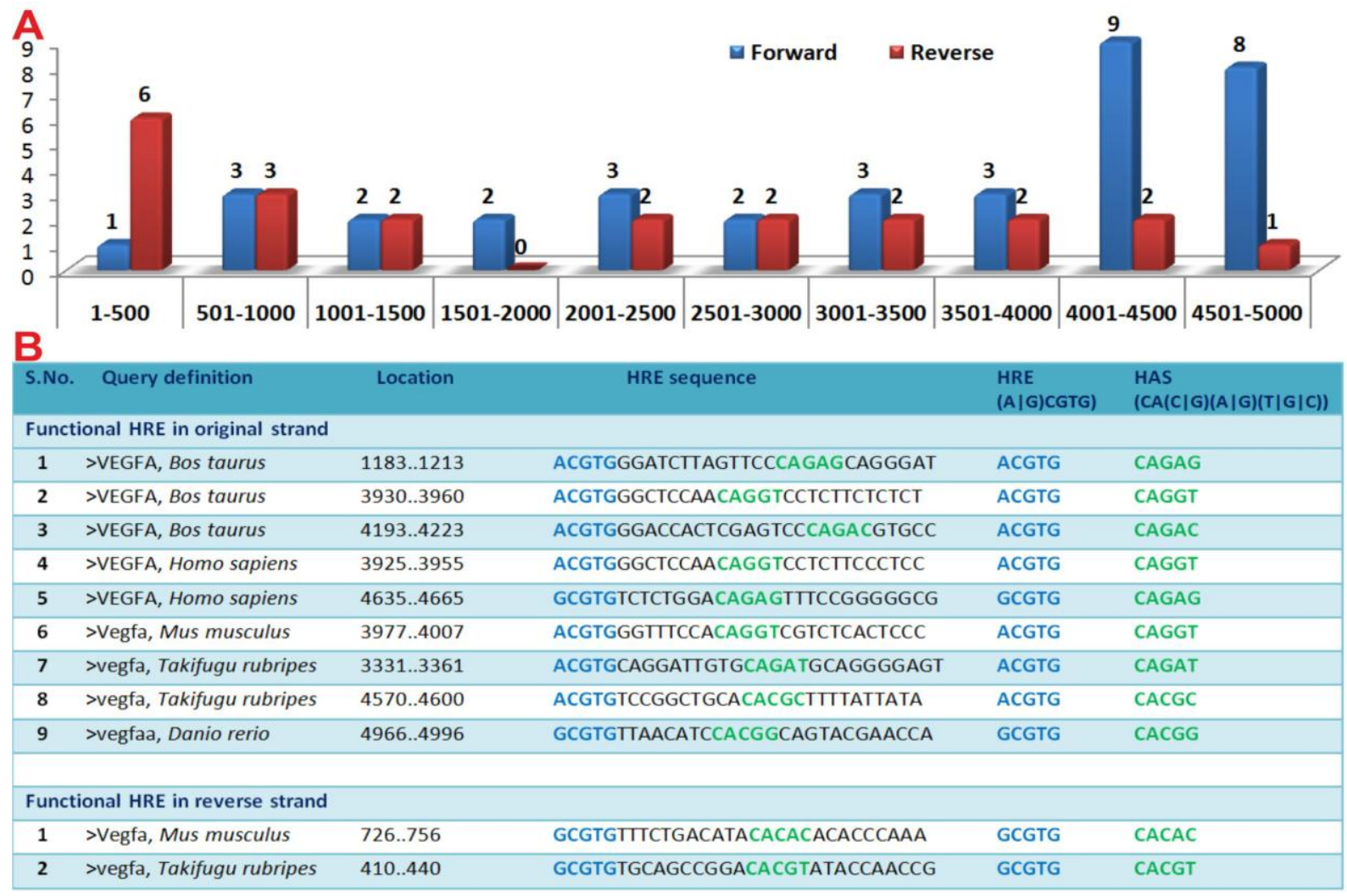


Further, many tools like Match (Kel et al., 2003) and ConTra (Hooghe et al., 2008) use PWMs library collected in the TRANSFAC (Wingender et al., 2001) and JASPAR (Bryne et al., 2008) databases for the identification of the TFBs in the query sequence. These databases do not provide availability nearly $80 \%$ of the scoring matrices of TFBs in the public release. On the other hand, several reports available on the hypoxia binding site and functional $\mathrm{HRE}$ in the upstream of hypoxia inducible genes in the vertebrates. Using this information in the pattern matching algorithm, HREFinder tool was developed specifically for functional HRE findings within a sequence or set of sequences, this tool also applicable for searching the canonical HRE in the hypoxia responsive genes in the genome-wide survey of vertebrates. This feature with specificity for finding the functional HRE in the upstream sequence of gene differentiates it from most other software tools that provide conserved site across a set of sequences.

\section{Conflict of interest}

The authors declare no conflict of interest

\section{Acknowledgment}

Authors are thankful to the Director, ICARNBFGR, Lucknow, for providing necessary facilities to carry out this work and to the CABin, ICAR-IASRI, New Delhi, for financial assistance. We are also thankful to Mrs. Reeta Chaturvedi and Mr. Ravi Kumar, ICAR-NBFGR, Lucknow, for providing assistance in an image preparation.

\section{References}

Bailey, T.L., Boden, M., Buske, F.A., Frith, M., Grant, C.E., Clementi, L., Ren, J., Li, W.W., Noble, W.S. 2009. MEME SUITE: tools for motif discovery and searching. Nucleic Acids Res., 37: W202-8.
Benita, Y., Kikuchi, H., Smith, A.D., Zhang, M.Q., Chung, D.C., Xavier, R.J. 2009. An integrative genomics approach identifies Hypoxia Inducible Factor-1 (HIF-1)target genes that form the core response to hypoxia. Nucleic Acids Res., 37: 4587602.

Boyer, R.S., Moore, J.S. 1977. A fast string searching algorithm. Communications of the ACM, 20: 762-772.

Bryne, J.C., Valen, E., Tang, M.H., Marstrand, T., Winther, O., da Piedade, I., Krogh, A., Lenhard, B., Sandelin, A. 2008 JASPAR, the open access database of transcription factor-binding profiles: new content and tools in the 2008 update. Nucleic Acids Res., 36: D102-106.

Fordel, E., Geuens, E., Dewilde, S., De Coen, W., Moens, L. 2004. Hypoxia/ischemia and the regulation of neuroglobin and cytoglobin expression. IUBMB Life, 56: 681-7.

Greenald, D., Jeyakani, J., Pelster, B., Sealy, I., Mathavan, S., van Eeden, F.J. 2015. Genome-wide mapping of Hif- $1 \alpha$ binding sites in zebrafish. BMC Genomics, 16: 923.

Guillemin, K., Krasnow, M.A. 1997. The hypoxic response: huffing and HIFing. Cell, 89: 9-12.

Hooghe, B., Hulpiau, P., van Roy, F., De Bleser, P. 2008. ConTra: a promoter alignment analysis tool for identification of transcription factor binding sites across species. Nucleic Acids Res., 36: W128-32.

Kajimura, S., Aida, K., Duan, C. 2006. Understanding hypoxia-induced gene expression in early development: in vitro and in vivo analysis of hypoxia-inducible factor 1-regulated zebra fish insulin-like growth factor binding protein 1 gene expression. Mol Cell Biol., 26: 1142-55.

Kel, A.E., Gössling, E., Reuter, I., Cheremushkin, E., Kel-Margoulis, O.V., Wingender, E. 2003. MATCH: A tool for searching transcription factor binding sites in DNA sequences. Nucleic Acids Res., 31: 3576-9. 
Kimura, H., Weisz, A., Ogura,,T, Hitomi, Y., Kurashima, Y., Hashimoto, K., D'Acquisto, F., Makuuchi, M., Esumi, H. 2001. Identification of hypoxia-inducible factor 1 ancillary sequence and its function in vascular endothelial growth factor gene induction by hypoxia and nitric oxide. J. Biol. Chem., 276: 2292-8.

Korhonen, J., Martinmäki, P., Pizzi, C., Rastas, P., Ukkonen, E. 2009. MOODS: fast search for position weight matrix matches in DNA sequences. Bioinformatics, 25: 3181-2.

Liu, Y., Cox, S.R., Morita, T., Kourembanas, S. 1995. Hypoxia regulates vascular endothelial growth factor gene expression in endothelial cells. Identification of a $5^{\prime}$ enhancer. Circ. Res., 77: 638-43.

Manalo, D.J., Rowan, A., Lavoie, T., Natarajan, L., Kelly, B.D., Ye, S.Q., Garcia, J.G., Semenza, G.L. 2005. Transcriptional regulation of vascular endothelial cell responses to hypoxia by HIF-1. Blood, 105: 659-69.

NCBI Resource Coordinators. 2017. Database Resources of the National Center for Biotechnology Information. Nucleic Acids Res., 45: D12-D17.

Ortiz-Barahona, A., Villar, D., Pescador, N., Amigo, J., del Peso, L. 2010. Genomewide identification of hypoxia-inducible factor binding sites and target genes by a probabilistic model integrating transcription-profiling data and in silico binding site prediction. Nucleic Acids Res., 38: 2332-45.
Rashid, I., Nagpure, N.S., Srivastava, P., Kumar, R., Pathak, A.K., Singh, M., Kushwaha, B. 2017. HRGFish: A database of hypoxia responsive genes in fishes. Sci Rep., 7: 42346.

Semenza, G.L. 2010. Defining the role of hypoxia-inducible factor 1 in cancer biology and therapeutics. Oncogene, 29: 625-34.

Smith, T.G., Brooks, J.T., Balanos, G.M., Lappin, T.R., Layton, D.M., Leedham, D.L., Liu, C., Maxwell, P.H., McMullin, M.F., McNamara, C.J., Percy, M.J., Pugh, C.W., Ratcliffe, P.J., Talbot, N.P., Treacy, M., Robbins, P.A. 2006. Mutation of von Hippel-Lindau tumour suppressor and human cardiopulmonary physiology. PLoS Med., 3(7): e290.

Van Rooijen, E., Santhakumar, K., Logister, I., Voest, E., Schulte-Merker, S., Giles, R., van Eeden, F. 2011. A zebrafish model for VHL and hypoxia signaling. Methods Cell Biol., 105: 163-90.

Wang, G.L., Semenza, G.L. 1995. Purification and characterization of hypoxia-inducible factor. J. Biol. Chem., 270: 1230-7.

Wenger, R.H., Stiehl, D.P., Camenisch, G. 2005. Integration of oxygen signaling at the consensus HRE. Sci. STKE., 2005: re12. Review.

Wingender, E., Chen, X., Fricke, E., Geffers, R.,Hehl, R., Liebich, I., Krull, M., Matys, V., Michae, H., Ohnhäuser, R., Prüss, M., Schacherer, F., Thiele, S., Urbach, S. 2001. The TRANSFAC system on gene expression regulation. Nucleic Acids Res., 29: 281-3.

\section{How to cite this article:}

Iliyas Rashid, Naresh Sahebrao Nagpure, Prachi Srivastava, Ajey Kumar Pathak, Ravindra Kumar, Vishwamitra Singh Baisvar, Mahender Singh and Basdeo Kushwaha. 2017. HRE Finder: A Tool for Quarrying Hypoxia-Response Element in Genomic Sequences. Int.J.Curr.Microbiol.App.Sci. 6(7): 1580-1586. doi: https://doi.org/10.20546/ijcmas.2017.607.190 